\title{
ELABORACIÓN DE UN MODELO EMPRESARIAL PARA QUE LAS PYME DEL SECTOR DE BEBIDAS Y ALIMENTOS LOGREN INCREMENTAR SU CICLO DE VIDA EN LA CIUDAD DE BOGOTÁ
}

\author{
DEVELOPMENT OF A BUSINESS MODEL SO THAT SMES IN THE FOOD AND \\ BEVERAGE SECTOR CAN INCREASE THEIR LIFE CYCLE IN THE CITY OF BOGOTÁ
}

Martín Felipe Cuevas Oviedo*
Erika Juliana Acevedo Solano**

Recibido: 17 de julio de 2017

Aceptado: 22 de septiembre de 2017

\section{Resumen}

El objetivo de este artículo es identificar y analizar los factores que permitieron a las PyME del sector alimentos y bebidas crecer y mantenerse en el mercado por un tiempo mayor a los cinco años, para así proponer una caracterización que sirva de referencia a otras empresas que estén empezando o que tengan problemas de sostenibilidad. Para ello, se utilizaron diferentes herramientas, como técnicas de observación, entrevistas a profundidad y técnicas estadísticas, para analizar los posibles factores que permiten la perdurabilidad de las PyME el en tiempo. En primera instancia, se realizó un análisis de la contribución de los diferentes subsectores nacionales al crecimiento de la producción en el sector industrial colombiano, en el que se pudo identificar al sector de alimentos y bebidas como el más representativo. Posteriormente, se estableció una muestra de las PyME de dicho sector, a las que se les realizó una entrevista de profundidad para identificar los factores claves de éxito. El resultado final es un modelo empresarial sustentable para que los emprendedores logren encaminar su organización a campos más competitivos, y que no solo se conformen con un equilibro entre sus ingresos y gastos, sino que puedan conseguir un crecimiento económico sustentable para alcanzar una competitividad en su sector y así superar la etapa de madurez en el ciclo de vida de una organización.

Palabras claves: PyME, factores claves de éxito, ciclo de vida, indicadores, modelo empresarial.

\footnotetext{
*Administrador empresas. Docente investigador, grupo de investigación CINDE, línea de investigación de Emprendimiento y Empresarismo, Fundación Universidad de América, Bogotá, Colombia. ORCID:http://orcid.org/00000003-0212-7622. martín.cuevas@investigadores.uamerica.edu.co

** Estudiante de Ingeniería Industrial. Coinvestigador, grupo de investigación CINDE, línea de investigación de Emprendimiento y Empresarismo, Fundación Universidad de América, Bogotá, Colombia. erika.acevedo@estudiantes.uamerica.edu.co
} 


\section{Abstract}

The objective of this article, is to identify and analyze the factors that allowed SMEs in the food and beverage sector to grow and stay in the market for a higher time than five years, to be able to propose a characterization that serves as a reference for other companies that are beginning, or which have problems of sustainability. For this purpose, different tools, such as observation techniques, in-depth interviews, and statistical techniques were used to analyze the possible factors that allow the durability of SMEs over time. In the first instance, an analysis of the contribution of the different national sub-sectors to the growth of production in the Colombian industrial sector was made, in which the food and beverage sector could be identified as the most representative. Subsequently, a sample of the SMEs of that industry was established, which were interviewed in depth to identify the key success factors. The end result is a sustainable business model so that entrepreneurs can route their organization to more competitive fields, and not only be conform to a balance of their incomes and expenses, but also, they can be able to get sustainable economic growth to achieve competitiveness in their sector and thus overcome the stage of maturity in the life cycle of an organization.

Keywords: SMEs, key success factors, life cycle, indicators, business model.

\section{INTRODUCCIÓN}

El objetivo de esta investigación es identificar y analizar los factores que permitieron a las PyME crecer y mantenerse en el mercado por un tiempo mayor a los cinco años. Esto con el fin de obtener una caracterización que sirva de referencia para otras empresas que estén empezando o que tengan problemas de sostenibilidad. Para lograr este objetivo, el presente trabajo de investigación utiliza distintas herramientas vistas en el Programa de Ingeniería Industrial para la recolección de información (pruebas, técnicas de observación, entrevistas a profundidad) y el análisis de datos (herramientas estadísticas) con el fin de reconocer los posibles factores que hacen que las PYMES perduren en el tiempo. Como primera medida, se identificaron parámetros de empresas exitosas que sirven de base para futuras decisiones de una organización. La importancia de identificar estos factores radica en que las PyME puedan tomar como referencia el modelo que surge de esta investigación. Esto con el fin de que las pequeñas y medianas empresas tengan una economía sólida, puedan crecer y ser más estables. Encontrar factores de éxito y buenas prácticas en las PyME podría cambiar la mentalidad de los emprendedores colombianos y de esta forma crear empresas que en un futuro sean exitosas. Para cumplir con este propósito, se planteó como objetivo general la elaboración de un modelo para que las PyME incrementen su ciclo de vida en el sector industrial, con base en el reconocimiento de buenas prácticas. El primer objetivo específico del proyecto fue identificar el subsector más representativo de las PyME localizadas en Bogotá, por medio de un análisis detallado de los subsectores que más le aportan a la producción del país. Posteriormente, se realiza un diagnóstico sobre las PyME del subsector de bebidas y alimentos, basado en el modelo de las cinco fuerzas de Porter y en la matriz DOFA. Este diagnóstico permite identificar los factores claves y las buenas prácticas que implementan las organizaciones en gestión estratégica y gerencial. Finalmente, se propone un modelo con factores de éxito (replicable a los diferentes subsectores de la economía) para lograr que una PyME perdure en el tiempo. 


\section{Marco té́rico}

Las empresas colombianas en su mayoría pertenecen al grupo de PyME y MiPyME. Esto indica que la economía colombiana está basada en micro, pequeñas y medianas empresas, siendo estas últimas las que aportan más del $60 \%$ de los empleos y mueven gran parte de la economía (Departamento Administrativo Nacional de Estadística, 2015). La situación actual de estas empresas es crítica, ya que la gran mayoría tiene que suspender sus actividades debido a que su rentabilidad, competitividad o productividad no les permite mantenerse en el mercado por más de cinco años. Esta situación se genera porque las empresas no tienen los recursos económicos, tecnológicos ni financieros para poder obtener una estabilidad en el sector en el que se encuentran, por lo que la vida de una empresa perteneciente a este grupo no es muy larga.

En Colombia existen aproximadamente veintitrés mil MiPyME, que se encuentran agrupadas principalmente en la ciudad de Bogotá. En esta ciudad están ubicadas alrededor del $96.4 \%$ de estas empresas, divididas por sectores económicos a lo largo de todo el territorio capitalino. El resto de las organizaciones se encuentra principalmente en Cali, Barranquilla y Bucaramanga (Cantillo, 12 de julio de 2011). Las pequeñas y medianas empresas son un factor fundamental para el crecimiento y desarrollo del país, así, pues, representan el $70 \%$ del empleo. Un $6 \%$ de los empleos en Bogotá son generados por microempresas, y alrededor del $40 \%$ de los salarios registrados por el país son originados por las PyME. E1 $45 \%$ de la producción de estas compañías está dedicada a la manufactura (Cantillo, 12 de julio de 2011).

E1 PIB de Colombia se distribuye por departamentos de la siguiente manera:

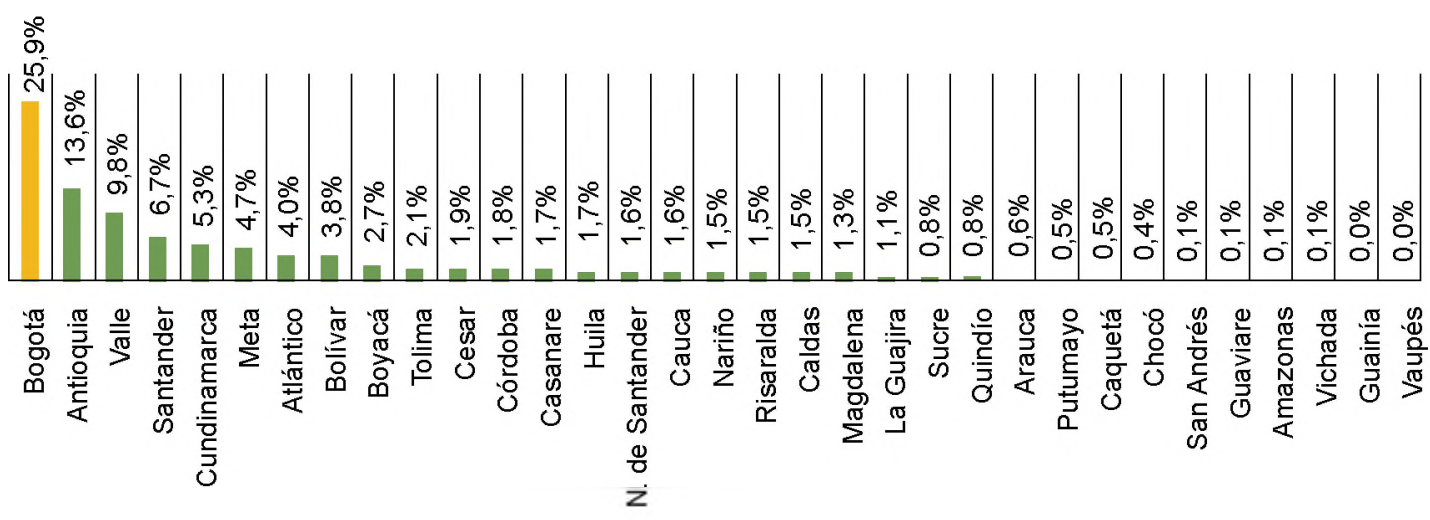

Figura 1. Distribución departamental del PIB en Colombia.

Fuente: Cámara de Comercio de Bogotá (2014).

Bogotá tiene el mayor porcentaje de participación del PIB en Colombia, así como se muestra en la figura 1 , lo que indica que la ciudad genera la mayor cantidad de empleo, empresas, negocios, competencias y demás factores asociados con la economía. Si bien esto es importante para la ciudad, también se evidencia una desigualdad en el desarrollo de las otras regiones, cuya participación es mínima. Con esto se puede inferir que los esfuerzos del gobierno se han centrado en pocos territorios y, por lo tanto, la calidad de vida en algunas zonas del país es muy baja, en comparación con departamentos con mayor participación (Antioquia, por ejemplo). De acuerdo a la distribución porcentual a nivel sectorial en Colombia, para el primer semestre del año 2014, los sectores industriales más destacados se presentan en la figura 2; según la figura, el subsector de alimentos y bebidas tuvo el mayor porcentaje de participación, con un $13 \%$, seguido por el sector de productos de caucho y plásticos. Este gráfico muestra la distribución de algunos de los sectores más representativos. 


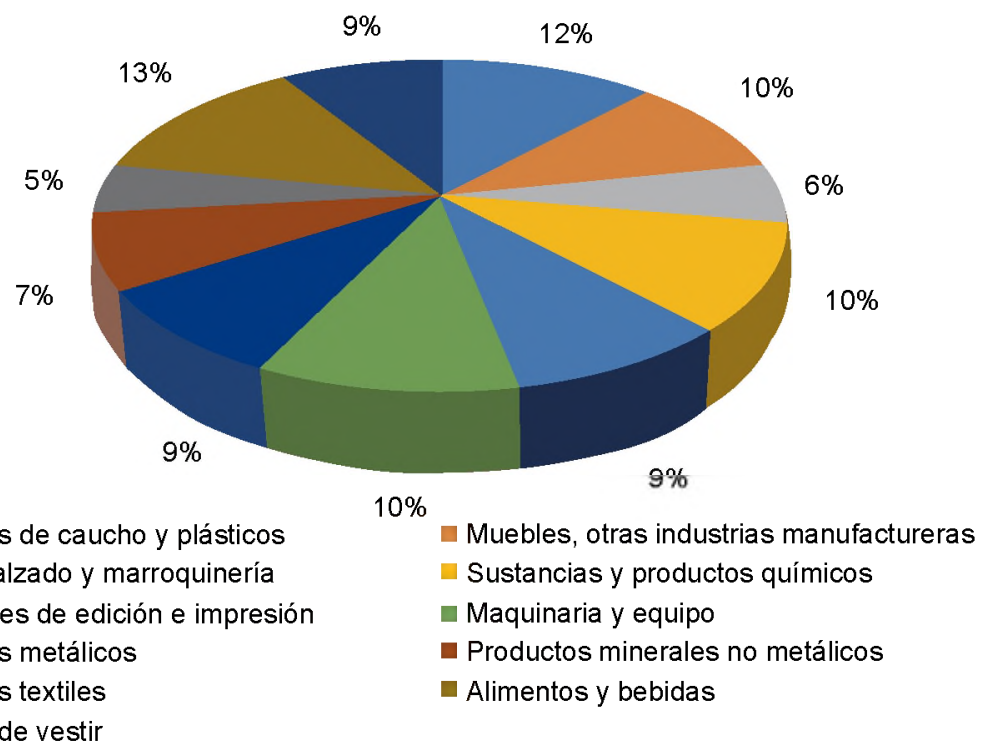

Figura 2. Distribución porcentual de la muestra a nivel sectorial.

Fuente: ANIF (2014).

En la figura 3 se puede observar la contribución de cada subsector al crecimiento de la producción en el sector industrial. Se visualiza como la mayor participación está en las industrias de alimentos para animales, transformación de madera (y sus productos) y la elaboración de cacao, chocolate y productos de confitería.

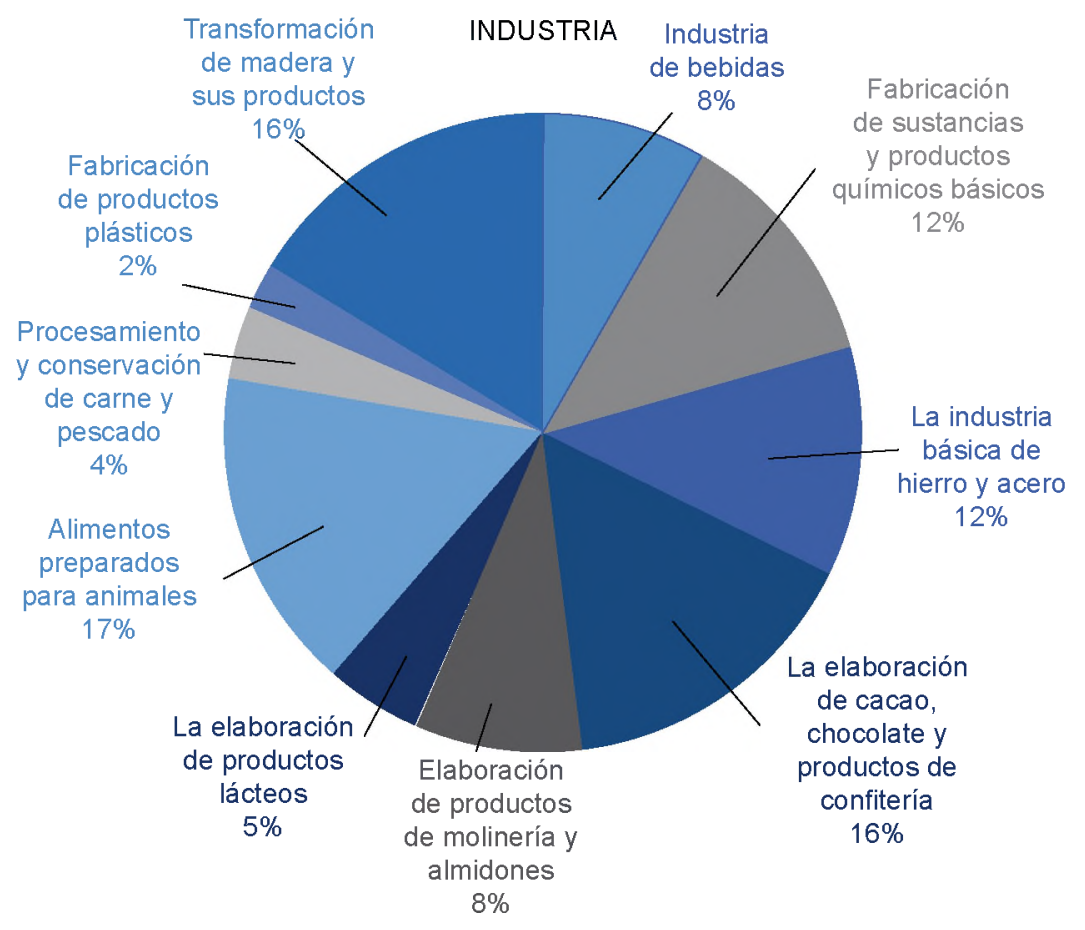

Figura 3. Distribución de la muestra a nivel sectorial enero-diciembre 2014.

Fuente: Ministerio de Industria y Comercio (2015). 
Uno de los sectores más representativos en la economía de nuestro país es el sector de alimentos y bebidas. En el 2014, las empresas pertenecientes a este sector registraron ingresos operacionales consolidados de $\$ 54$ billones, lo que significó un aumento del $10 \%$ frente al año anterior y un incremento de $11 \%$ de los ingresos operacionales. Los activos, pasivos y patrimonio generaron un aumento alrededor del $9 \%$. El mayor aumento se presentó en las ganancias, con un $17.7 \%$; de estas cifras se puede concluir que el crecimiento fue mínimo, pero se observa que, aun así, la generación de ganancias se incrementa en un porcentaje significativo (Superintendencia de Sociedades, 2015). El incremento se debe a que los colombianos tienen mayor poder adquisitivo y esto ha permitido el esparcimiento de la base del consumo, lo que genera una mayor demanda.

\section{Subsector de alimentos y bebidas}

Después de la crisis 2010-2012, la industria colombiana inició su recuperación con los sectores más dinámicos de la industria, como es el caso del sector de alimentos, con un $6 \%$, los productos lácteos, con un $7.9 \%$, y otros productos alimenticios, con un $5.4 \%$. En el 2014 se presentó un impulso de factores, como un incremento en la producción de productos agropecuarios, la disminución de problemas de orden público y un clima más estable en la región. La industria de alimentos y bebidas mantiene la mayor distribución de las industrias y la más alta participación en el PIB. Para esta industria se espera una demanda creciente de productos como cacao, chocolate, confitería, alimentos para mascotas y bebidas. Esta industria es atractiva, ya que el crecimiento de la población y los diferentes acuerdos económicos hacen que sea un sector en constante desarrollo e innovación. La mayor parte de los productos de este sector se consumen en Bogotá y regiones aledañas. Bogotá es también la principal productora de alimentos y bebidas del país. Esta investigación escogió este subsector para investigarlo, analizarlo y encontrar un modelo de buenas prácticas en la industria de alimentos y bebidas.

\section{Análisis de las cinco fuerzas de Porter para el subsector de alimentos y bebidas}

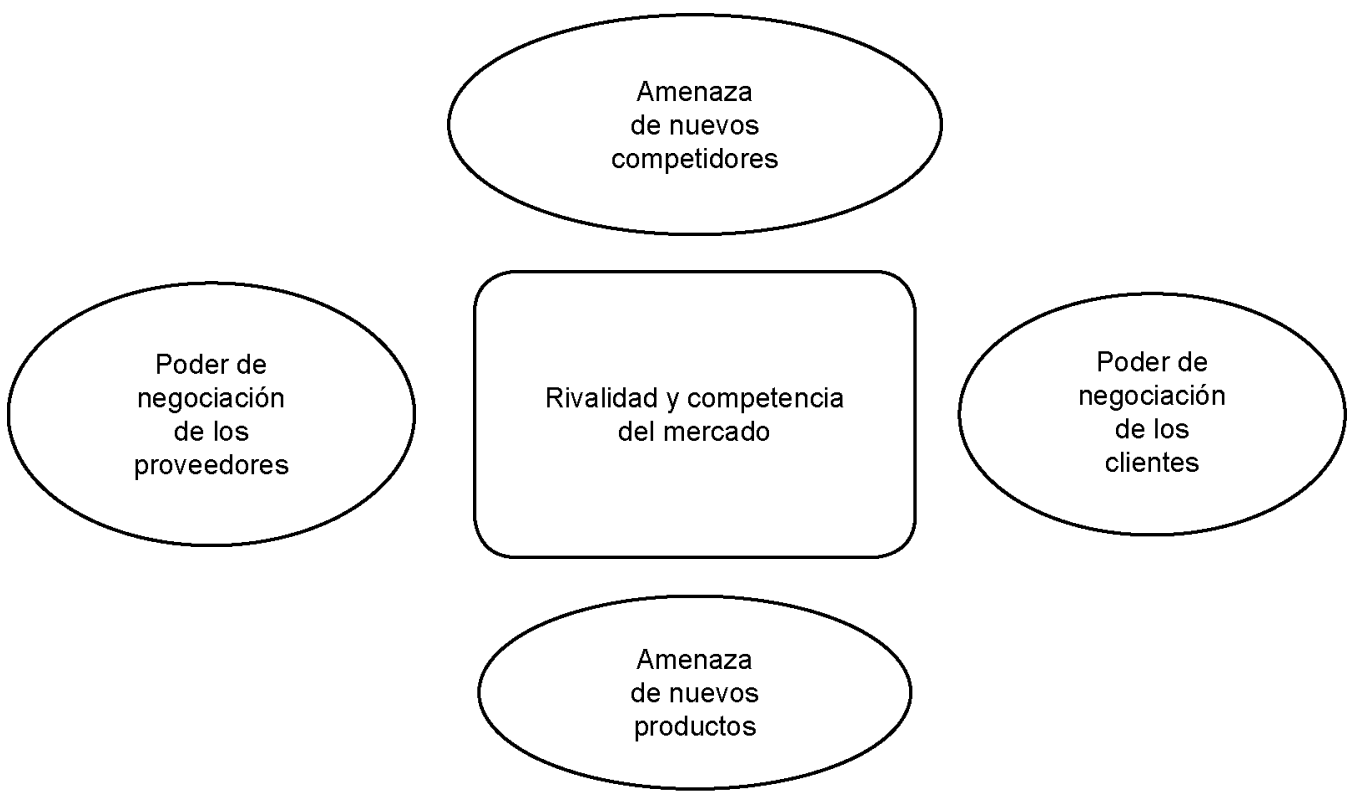

Figura 4. Análisis de las cinco fuerzas de Porter. 
Según el análisis de las cinco fuerzas de Porter para el sector de alimentos y bebidas, se puede afirmar que existen altas barreras de ingreso al capital, bien sea en infraestructura o en recursos humanos. El mercado es dinámico en el sector, especialmente con las marcas de alto reconocimiento, ya que estas se asocian con buena calidad y esto puede afectar en gran medida al crecimiento de una nueva empresa. También existen clientes insatisfechos que buscan alimentos más saludables y menos procesados, lo que puede generar un mercado potencial. Si bien el poder de negociación para una nueva empresa pequeña no es muy amplio, esto depende principalmente del producto que se esté fabricando, puesto que en otros casos existe gran variedad de proveedores, lo que disminuye su poder.

Gracias a la variedad de productos que ofrece el mercado, el poder de negociación de los clientes es alta, pues estos pueden cambiar fácilmente a cualquier otro producto. También existe la posibilidad de la entrada de nuevos productos, pues en la actualidad la fidelidad a una marca se ha reducido y los consumidores tienden a buscar productos diferentes. Para las empresas que están en este sector existen otras dificultades relacionadas con el alto costo de la producción, como los empaques y las maquinarias.

Como en todo sector, existe una alta competencia, pero como consecuencia de la su recuperación actual, esta ha disminuido un poco. Puede que en cierta parte el sector no vea tan atractivo el ingreso de nuevas empresas, aunque existen diferentes nichos de mercados sin explorar, como los alimentos saludables, orgánicos y artesanales, entre otros, que no presentan barreras tan altas para su ingreso y tienen cada vez más consumidores. Hay productos que por su variedad están saturados, pero otros que aún están en sus inicios pueden tener nuevos competidores.

\section{Análisis DOFA en el subsector de alimentos y bebidas}

En la tabla 1 se presenta el cruce de la matriz DOFA y sus estrategias para el subsector de alimentos y bebidas:

Tabla 1. Análisis matriz DOFA

\begin{tabular}{|c|c|c|}
\hline & FORTALEZAS & DEBILIDADES \\
\hline & 1. Crecimiento en el sector & $\begin{array}{l}\text { 1. Conocimiento insuficiente para } \\
\text { algunos procesos }\end{array}$ \\
\hline & 2. Estándares de clase mundial en bebidas & 2. Poca eficiencia en el sector \\
\hline & 3. Producción de productos de calidad & 3. Poca inversión \\
\hline & 4. Crecimiento en la fabricación a terceros & 4. Precios no competitivos \\
\hline & $\begin{array}{l}\text { 5. Tecnología de punta en algunos } \\
\text { subsectores }\end{array}$ & 5. Altos costos de distribución \\
\hline & 6. Variedad de productos & 6. Falta innovación y desarrollo \\
\hline OPORTUNIDADES & Estrategias FO & Estrategias DO \\
\hline $\begin{array}{l}1 \text { Crecimiento de los productos } \\
\text { de la canasta }\end{array}$ & 1. 1 Desarrollar productos & $\begin{array}{l}1.4 \text { Implementar capacitaciones de } \\
\text { producción }\end{array}$ \\
\hline $\begin{array}{l}\text { 2. Tendencias de innovación y } \\
\text { funcionalidad }\end{array}$ & $\begin{array}{l}\text { 2.3 Mantener y fortalecer la calidad en } \\
\text { nuevos productos }\end{array}$ & $\begin{array}{l}\text { 2.2 Prestar funciones } \\
\text { complementarias, diversificación } \\
\text { concéntrica }\end{array}$ \\
\hline $\begin{array}{l}\text { 3. Crecimiento de productos } \\
\text { saludables }\end{array}$ & 3.6 Crear nuevas presentaciones & $\begin{array}{l}\text { 3.5 Especializar la empresa en } \\
\text { productos y procesos }\end{array}$ \\
\hline
\end{tabular}




\begin{tabular}{|c|c|c|c|}
\hline OPORTUNIDADES & & Estrategias FO & Estrategias DO \\
\hline $\begin{array}{l}\text { 4. Desarrollo de nuevos } \\
\text { productos lácteos }\end{array}$ & 4.5 & $\begin{array}{l}\text { Especializar la empresa en algunos } \\
\text { productos }\end{array}$ & $\begin{array}{l}\text { Desarrollar promoción y } \\
\text { publicidad }\end{array}$ \\
\hline 5. Clientes no satisfechos & 6.4 & Desarrollar de mercados & $\begin{array}{l}\text { Desarrollar nuevas áreas } \\
\text { geográficas }\end{array}$ \\
\hline \multicolumn{4}{|l|}{$\begin{array}{l}\text { 6. Crecimiento en } \\
\text { exportaciones. }\end{array}$} \\
\hline AMENAZAS & & Estrategias FA & Estrategias DA \\
\hline $\begin{array}{l}\text { 1. Presencia alta de } \\
\text { contrabando }\end{array}$ & 1.1 & $\begin{array}{l}\text { Presentar productos ajustables al } \\
\text { mercado }\end{array}$ & $\begin{array}{l}\text { 3.1 Implementar investigación para } \\
\text { ampliar conocimiento }\end{array}$ \\
\hline $\begin{array}{l}\text { 2. Inestabilidad en la tasa de } \\
\text { cambio }\end{array}$ & 3.4 & Penetrar mercados & $\begin{array}{l}\text { Integrar áreas para reducir } \\
\text { costos }\end{array}$ \\
\hline 3. Baja demanda interna & 4.6 & $\begin{array}{l}\text { Plantear publicidad de productos de } \\
\text { clase mundial }\end{array}$ & $\begin{array}{l}\text { Desarrollar producto con } \\
\text { inversión de investigación y } \\
\text { desarrollo }\end{array}$ \\
\hline $\begin{array}{l}\text { 4. Gran variedad de } \\
\text { importaciones }\end{array}$ & 6.2 & Desarrollar nuevos mercados & $\begin{array}{l}\text { Formalizar legalmente la } \\
\text { empresa }\end{array}$ \\
\hline $\begin{array}{l}\text { 5. Crisis con relaciones con } \\
\text { países vecinos }\end{array}$ & 8.5 & Aumentar los índices de productividad & $\begin{array}{l}\text { Realizar unión empresarial para } \\
\text { acuerdos gubernamentales }\end{array}$ \\
\hline 6. Acuerdos económicos & 8.3 & Certificar en normas de calidad & $\begin{array}{l}\text { Promover la inversión y } \\
\text { beneficios en el desarrollo }\end{array}$ \\
\hline 7. Aumento de carga tributaria & & & \\
\hline 8. Baja inversión extranjera & & & \\
\hline
\end{tabular}

En la figura 5 se puede observar diversas estrategias para afrontar las diferentes dificultades que presenta el subsector en estudio.

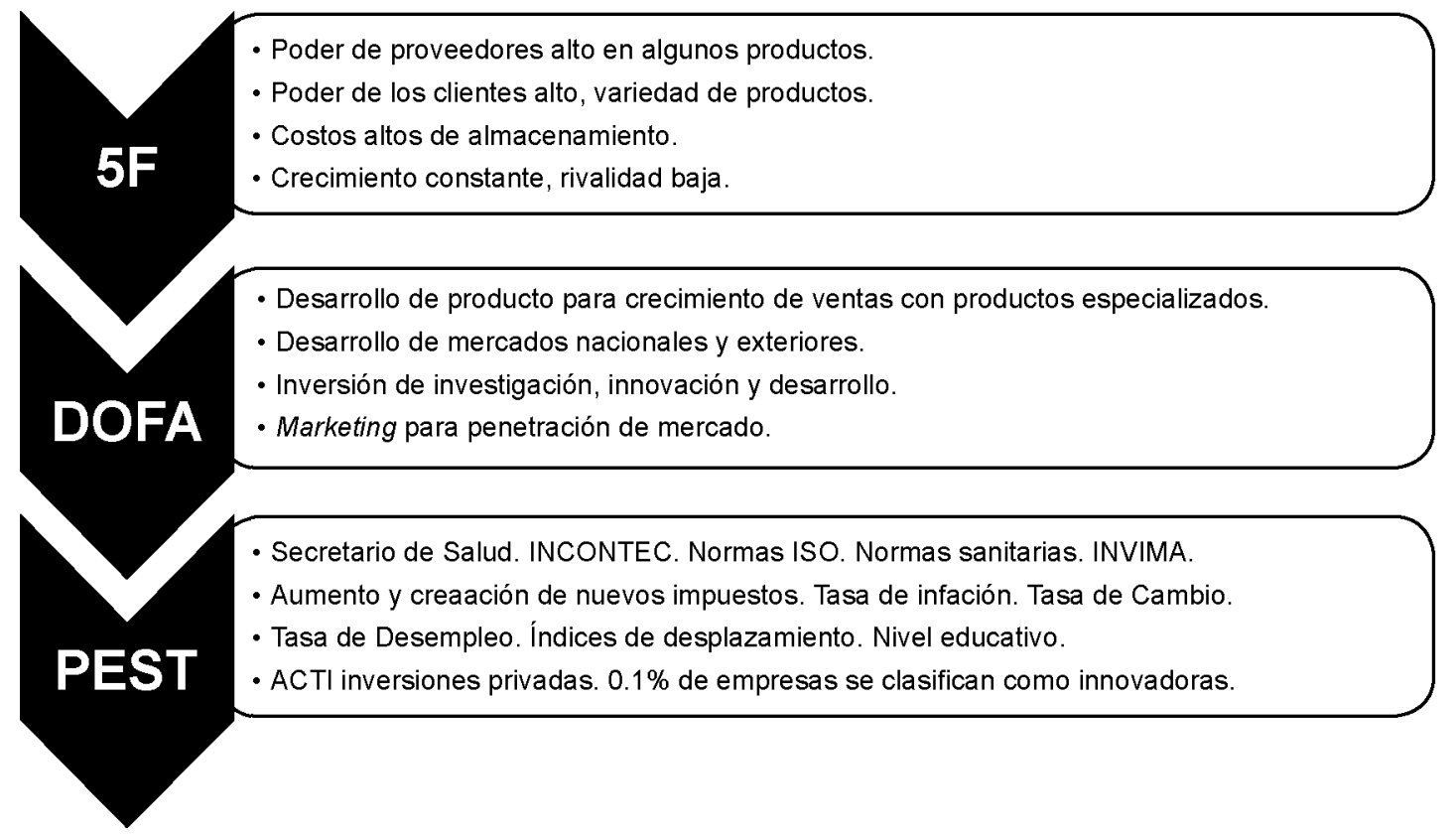

Figura 5. Resumen sector de alimentos y bebidas. 
Una de ellas es el desarrollo de productos para el crecimiento en las ventas, por lo que se mejora y especializa los productos e invierte en la innovación y desarrollo, para de esta manera aprovechar las oportunidades que ofrece el sector. Con la estrategia de desarrollo de mercado se pretende introducir el producto a otras áreas geográficas, ya sean mercados nacionales o extranjeros, aprovechando los diferentes tratados y así tener un equilibrio. Para lograr que toda empresa y sus respectivos productos tengan una acogida, es necesario introducir una estrategia de marketing que logre penetrar el mercado y pueda incrementar su participación. La estrategia es la herramienta fundamental para cumplir con los objetivos y metas de una organización, en los que cada empresa presenta diferencias con las que se puede montar la planeación de las decisiones a seguir.

\section{MÉTodo}

El desarrollo de la investigación se realizó desde un enfoque de tipo descriptivo-explicativo, ya que se busca describir y explicar las variables que afectan a las PyME bogotanas en cuanto a su perdurabilidad. Asimismo, por medio de herramientas cuantitativas se busca encontrar las causas del fenómeno. Esta investigación aplicó y ejecutó un tipo de investigación descriptivo y explicativo por medio de estudio de casos y estudios correlaciónales, con el fin de resolver el problema de la perdurabilidad de las PyME bogotanas.

El tipo de muestreo fue probabilístico con muestreo estratificado. Con esto se pretende identificar si existe alguna diferencia entre las empresas de alimentos o las empresas de bebidas. Se dividieron los grupos de acuerdo al número de empresas a investigar. En este caso, el subgrupo número 1 corresponde al grupo de alimentos (326 empresas), y el subgrupo 2 , al grupo de bebidas ( 28 empresas). Debido a que no se pudo acceder a una base de datos de todas las organizaciones de esta población, se investigaron las empresas a las que se tuvo acceso más cercano y facilidad en la obtención de información.

\section{Tamaño de la muestra}

Ya que la población objetivo es finita, establecemos que:

$$
n=\frac{N^{*} z^{2} * p^{*} q}{E^{2} *(N-1)+z^{2} * p^{*} q}
$$

Dónde:

$\mathrm{N}=$ tamaño de la muestra que deseamos determinar o saber.

$\mathrm{z}=$ grado de confianza para obtener resultados seguros $\mathrm{o}$ adecuados en el procedimiento de determinar un tamaño de muestra que sea representativa.

$\mathrm{N}=354$

$Z=95 \%$, entonces, $95 \%$ entre $2=47.5 \%$ entre $100=0.475=Z=1.96$.

$\mathrm{E}=5 \%$, entonces, $5 \%$ entre $100=0.05$.

$\mathrm{p}=50 \%$, entonces; $50 \%$ entre $100=0.5$.

$\mathrm{q}=50 \%$, entonces; $50 \%$ entre $100=0.5$.

Por lo tanto:

$\mathrm{p}=0.05$

$\mathrm{q}=0.05$

$\mathrm{E}=0.05$

$\mathrm{N}=354$ 


$$
\begin{gathered}
n=\frac{354 * 1.96^{2} * 0.05 * 0.05}{0.05^{2} *(354-1)+1.96^{2} * 0.05 * 0.05} \\
n=3.81
\end{gathered}
$$

Por lo tanto, la muestra arroja un resultado de cuatro entrevistas, que se realizaron por afijación proporcional de acuerdo a los dos subgrupos identificados. Sin embargo, para una mayor aproximación en los resultados, se tomaron un total de ocho entrevistas: cinco empresas del sector alimentos y tres empresas del sector bebidas.

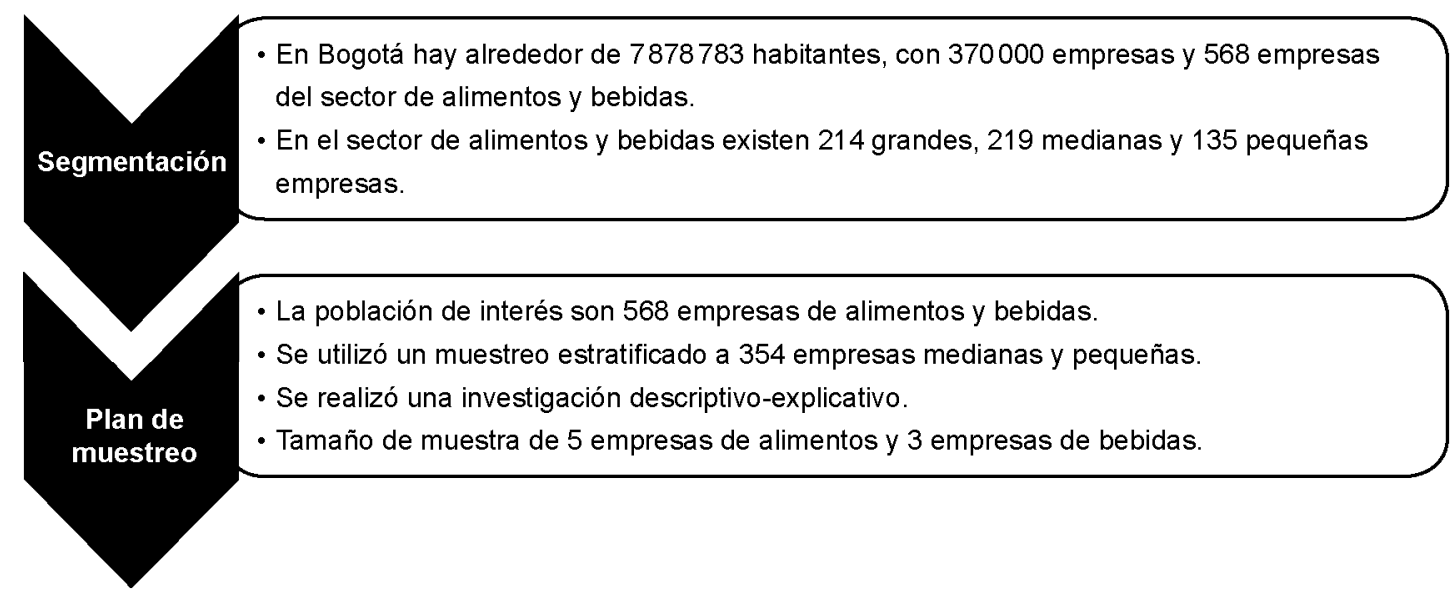

Figura 6. Plan de Muestreo

\section{Resultados}

\section{Principales hallazgos de las entrevistas a profundidad realizadas}

- La mayoría de las empresas que se entrevistaron se encuentran entre los 6 y los 9 años de vida. Esto quiere decir que ya pasaron la barrera de los 5 años, tiempo en el que la mayoría de las organizaciones no logran sobrevivir.

- $\quad$ E1 $70 \%$ de los encuestados contestaron que su idea de negocio surgió por emprendimiento; factor que es muy importante para estabilizar la economía de un país, debido a que se necesitan ideas para que surjan cambios.

- Las tres estrategias más importantes para las organizaciones son la competitividad, la innovación y el desarrollo de producto, cada una con un $20 \%$ de participación.

- Las empresas generan sus estrategias de acuerdo al plan estratégico que tengan; estas se implementan por medio de un análisis de la competencia, un análisis interno y externo y basándose en herramientas que generan información para la toma de decisiones.

- El desempeño de la organización refleja su estado. Las empresas entrevistadas coincidieron que los aspectos más importantes son la calidad y el reconocimiento; estos dos aspectos están relacionados entre sí, puesto que el reconocimiento lo genera el producto, y si este satisface de manera satisfactoria las necesidades del consumidor, se relaciona con la calidad.

- Uno de los aspectos en los que no se puede fallar es la calidad, ya que se puede incurrir en problemas legales o de cualquier otra índole. Otro factor determinante es tener una buena relación con los clientes y generar una atención óptima, rápida y de alta calidad. 
- Las principales dificultades a las que se enfrentan las organizaciones son la competencia en el mercado, la ilegalidad en los productos y la falta de solvencia económica para la adquisición de insumos, herramientas y maquinaria, recurso humano, publicidad y mercadeo.

- Existen varios problemas para mantener una organización en un mercado, relacionados con productividad, competitividad, rivalidad, poco crecimiento, entre otras.

- Las certificaciones de calidad ayudan a cerrar contratos y crean lazos con los proveedores y clientes.

- Los factores clave son calidad del producto, atención al cliente (los clientes son los motores de la industria), disciplina en los procesos, personal capacitado y comprometido con la empresa y manejo administrativo responsable y ético.

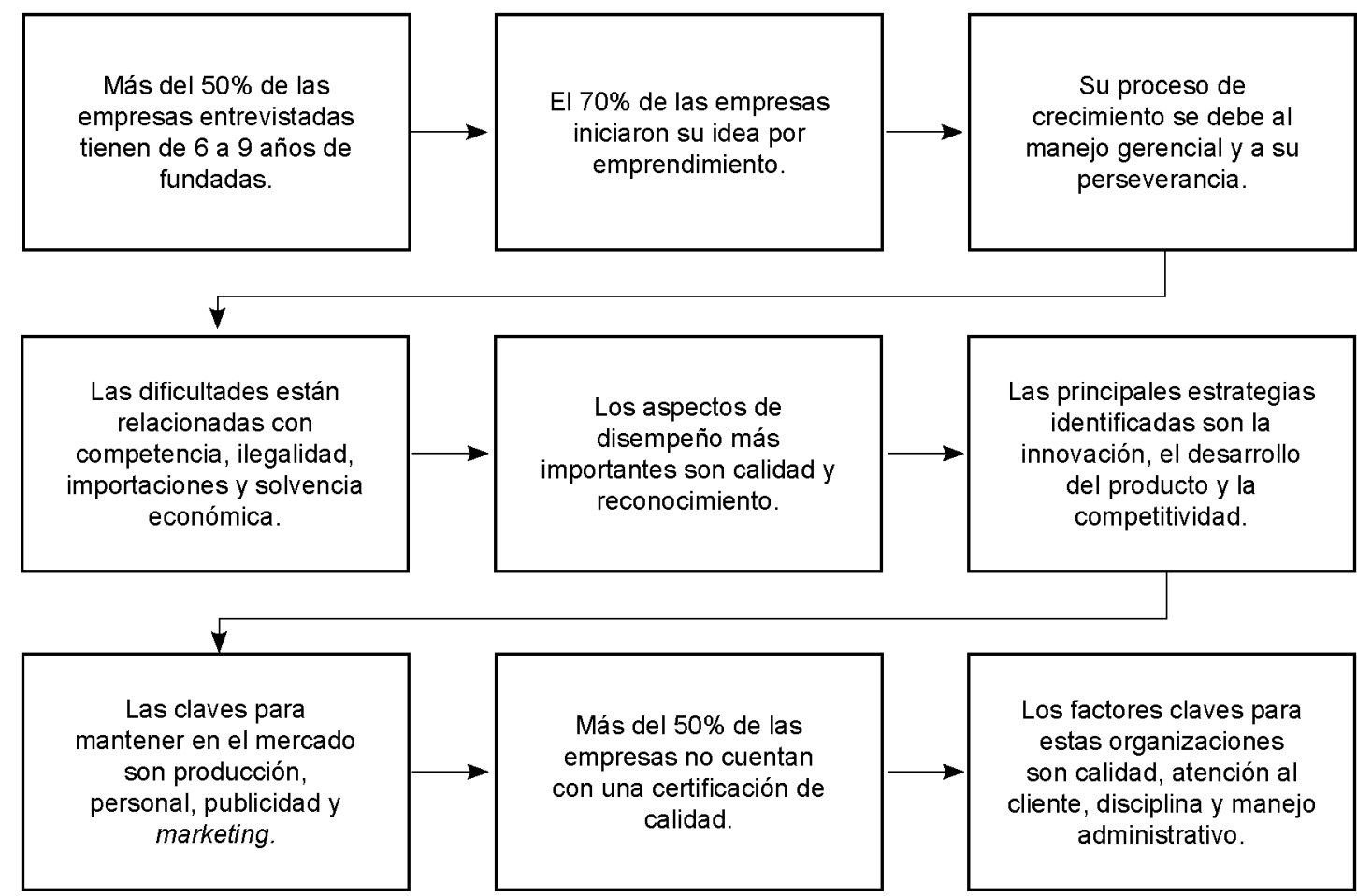

Figura 7. Principales hallazgos.

\section{Elaboración del modelo de factores claves para PyME}

A partir de los hallazgos identificados en las entrevistas realizadas a las empresas del sector de bebidas y alimentos, en la figura 8 se presenta el modelo conceptual de desarrollo empresarial basado en competencias. Con este modelo se busca dar pautas para que cada entidad aprenda a usarlo y adaptarlo a su propio entorno (el aprendizaje de la empresa debe ser continuo). La primera etapa de este modelo es el desarrollo de una mentalidad empresarial; la segunda, la identificación y evaluación de ideas de negocio; la tercera, enfatizar en la estructura y evaluación de la oportunidad de negocio; la cuarta, la elaboración de un plan de negocio integral; y la quinta, el proceso de arranque. Estas etapas deben estar ajustadas a los recursos que disponga cada empresa y se deben basarse en competencias personales y competencias de conocimiento, para así generar un empresario integral en el contexto empresarial. Este modelo permite realizar la evaluación, base de toma de decisiones 
y retroalimentación, por medio de mecanismos de seguimiento y el desarrollo de las competencias de cada uno de los miembros de la organización.

A continuación, se presentan algunos modelos para la elaboración de un modelo empresarial de factores claves de éxito, en los que se encontraron diferentes factores con los que las empresas han logrado mantenerse en el mercado.

\section{Modelos tipo de factores claves de éxito}

Los diferentes modelos estratégicos de un negocio se basan principalmente en las herramientas que definen el negocio y su planeación, por lo que los factores claves de éxito tienen una importancia vital para la planeación de sus metas. El modelo de John F. Rockart (1980), teórico en el comportamiento organizacional, define a los factores claves de éxito como:

Número limitado de áreas en las cuales las obtenciones de resultados satisfactorios asegurarán un rendimiento competitivo para el individuo, el departamento o la organización. Son áreas donde las cosas deben ir bien para que el negocio pueda alcanzar y mantener sus metas establecidas. (p. 7)

E1 modelo de negocio Canvas, creado por Alexander Osterwalder, incluye todos los aspectos relacionados con la organización, desde clientes y socios clave, hasta la estructura de costos. En este modelo se estipulan pautas para atraer clientes, definir ofertas e implementar estrategias de publicidad.

El modelo de Russel Ackoff evidencia la importancia de la planeación interactiva, pues cuando no se presenta una planeación se genera una afectación en la organización. Este modelo resalta el análisis y la compresión de los cambios en forma global, la constante actualización con el entorno y la planeación con la que se va hacer frente a estos cambios. En el modelo de Ackoff se observa la importancia de relacionar la organización con la estrategia de planeación y la volatilidad que puede tener el entorno, así como el grado de afectación de esta hacia la organización.

Ackof establece tres principios de la planeación interactiva:

- Principio participio: solo con la participación de los miembros de la organización se puede realizar el proceso y desarrollar la planeación.

- Principio de continuidad: existen diferentes variables que afectan la realización de la planeación, por ello se debe tener una permanente observación y control.

- Principios holísticos: se debe coordinar e integrar la planeación de manera simultánea para obtener mejores resultados (Sesento, 2016).

Finalmente, el modelo de Fred David se basa en tres etapas: formulación, ejecución y evaluación. Este modelo se enfoca en la toma de decisiones de la organización por medio de información dada cuantitativamente y cualitativamente frente a diferentes circunstancias de incertidumbre. Inicia con la identificación de la misión, objetivos y estrategias de la organización antes de realizar el proceso de planeación estratégica; posteriormente, se requiere de una auditoria externa e interna para identificar fortalezas, amenazas, oportunidades y debilidades. Con esta información recolectada se puede realizar una nueva misión de la organización (Estrategias, 2016).

Los modelos citados anteriormente son la base para la realización del modelo empresarial de factores claves de éxito propuesto en el presente artículo. Esta propuesta se puede considerar como un modelo estratégico debido al comportamiento que tienen los factores en la organización: la planeación, ejecución y evaluación de todas las estrategias, y el buen funcionamiento de la organización. 


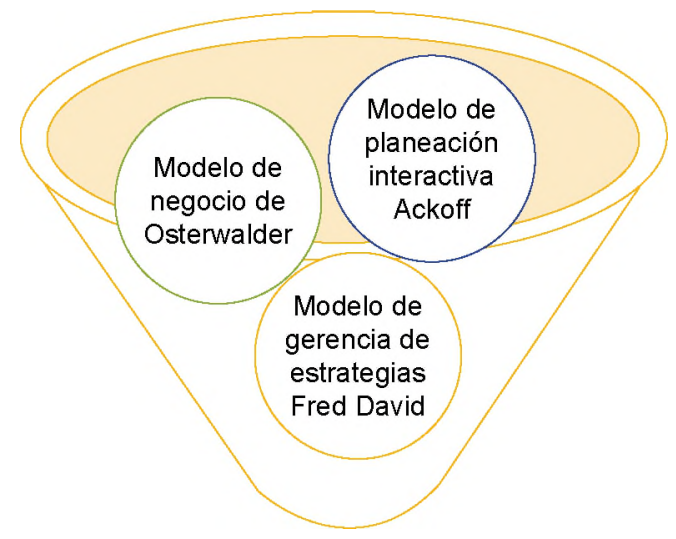

Figura 8. Modelo de factores claves de éxito

\section{Modelo empresarial de factores claves de éxito}

Tomando como base los modelos anteriores, sumado a la investigación realizada, se elaboró un modelo basado en el ciclo de vida de la organización. Este modelo inicia con el plan de negocio y el plan estratégico, seguidos por la participación, la identificación, la retroalimentación y el control en el interior de la organización.

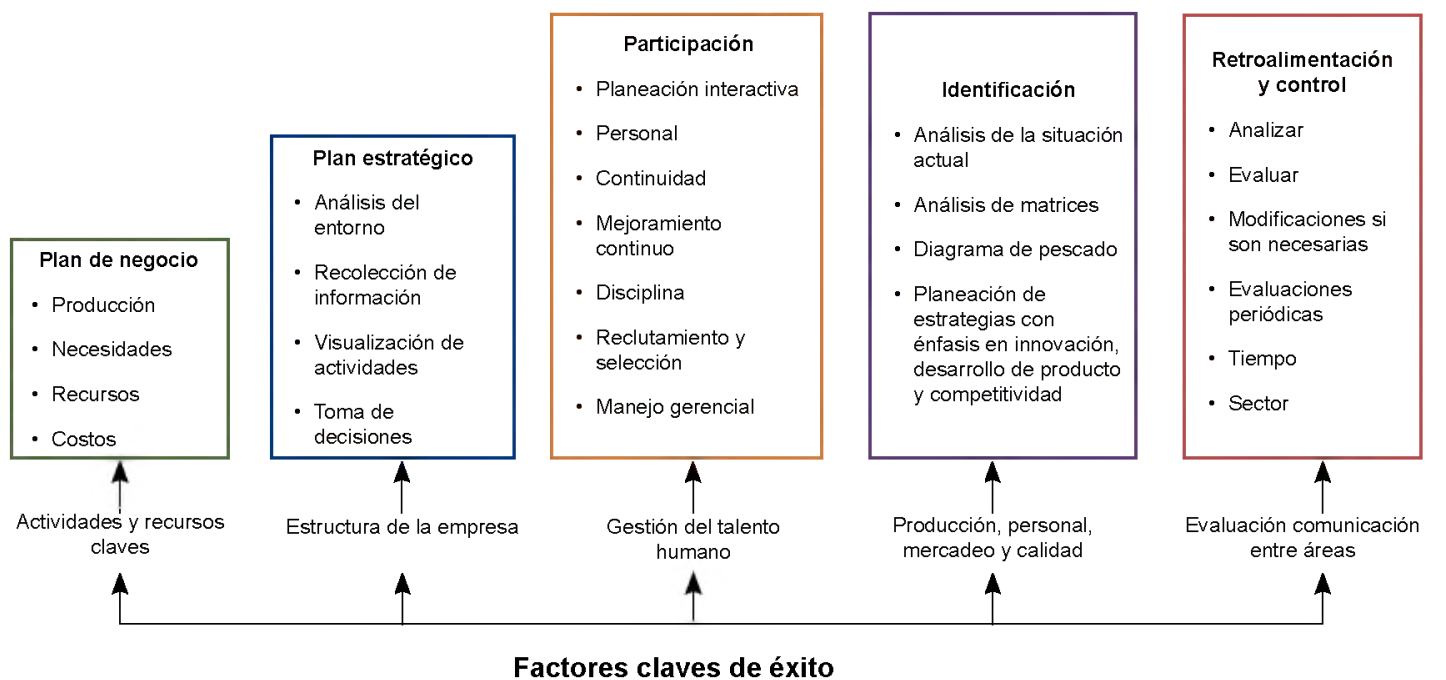

Figura 9. Modelo de factores claves de éxito. 


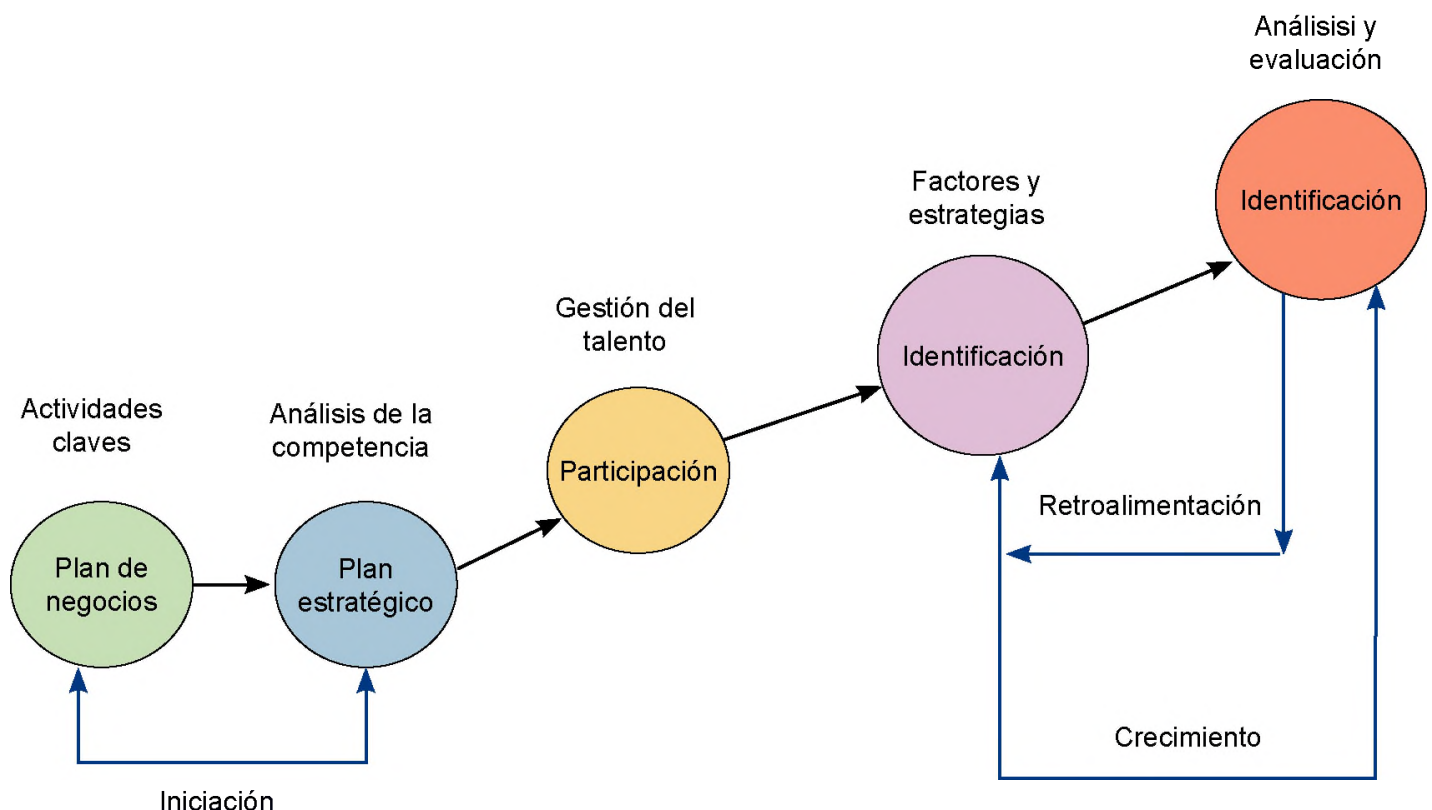

Figura 10. Generalidades del modelo

Este modelo propuesto se divide en dos etapas: etapa de iniciación y etapa de crecimiento. En estas dos etapas se incluyen los factores que se deben tener en cuenta para que la empresa logre incrementar su ciclo de vida; si bien no son los únicos, sí son los principales según la investigación realizada.

La primera etapa cuenta con dos divisiones: el plan de negocios, en la que se presenta un estudio de factibilidad, y la realización de un plan de negocios, en la que se identifican las actividades y recursos claves del proyecto. También se realiza un plan estratégico, en el que se recomienda realizar una recolección de datos y un análisis de la competencia, para posteriormente desarrollar el diseño de estructura y el modelo de ingresos.

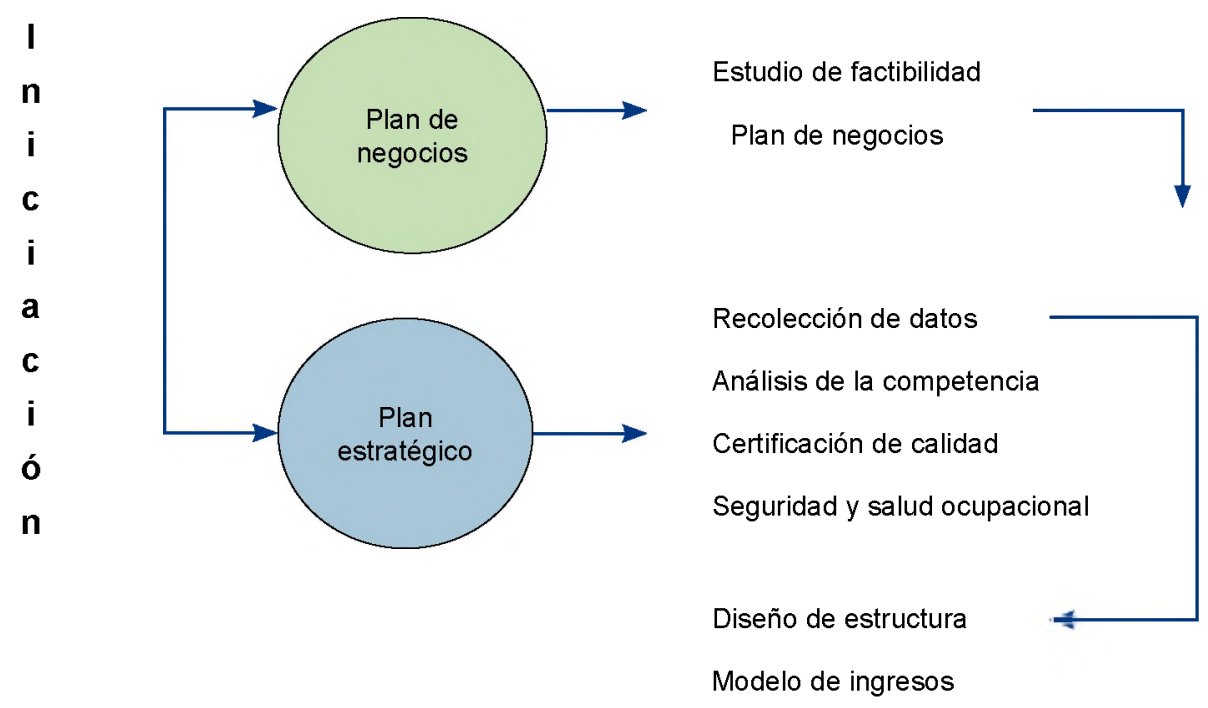

Figura 11. Modelo de factores claves de éxito: etapa de iniciación. 
La etapa de creación tiene tres divisiones: participación, identificación y control. La participación se basa en la disciplina y perseverancia de la organización frente a su ejecución del plan estratégico, que se debe enfocar en la divulgación y en el manejo del personal por medio de la gestión del talento humano. En la identificación se busca encontrar los aspectos y los factores claves y estrategias que la organización debe controlar para disminuir los riesgos organizacionales. En la etapa de control se analiza y evalúa las anteriores estrategias y el funcionamiento de la organización, retroalimentando los resultados para realizar una nueva planeación en un tiempo específico.

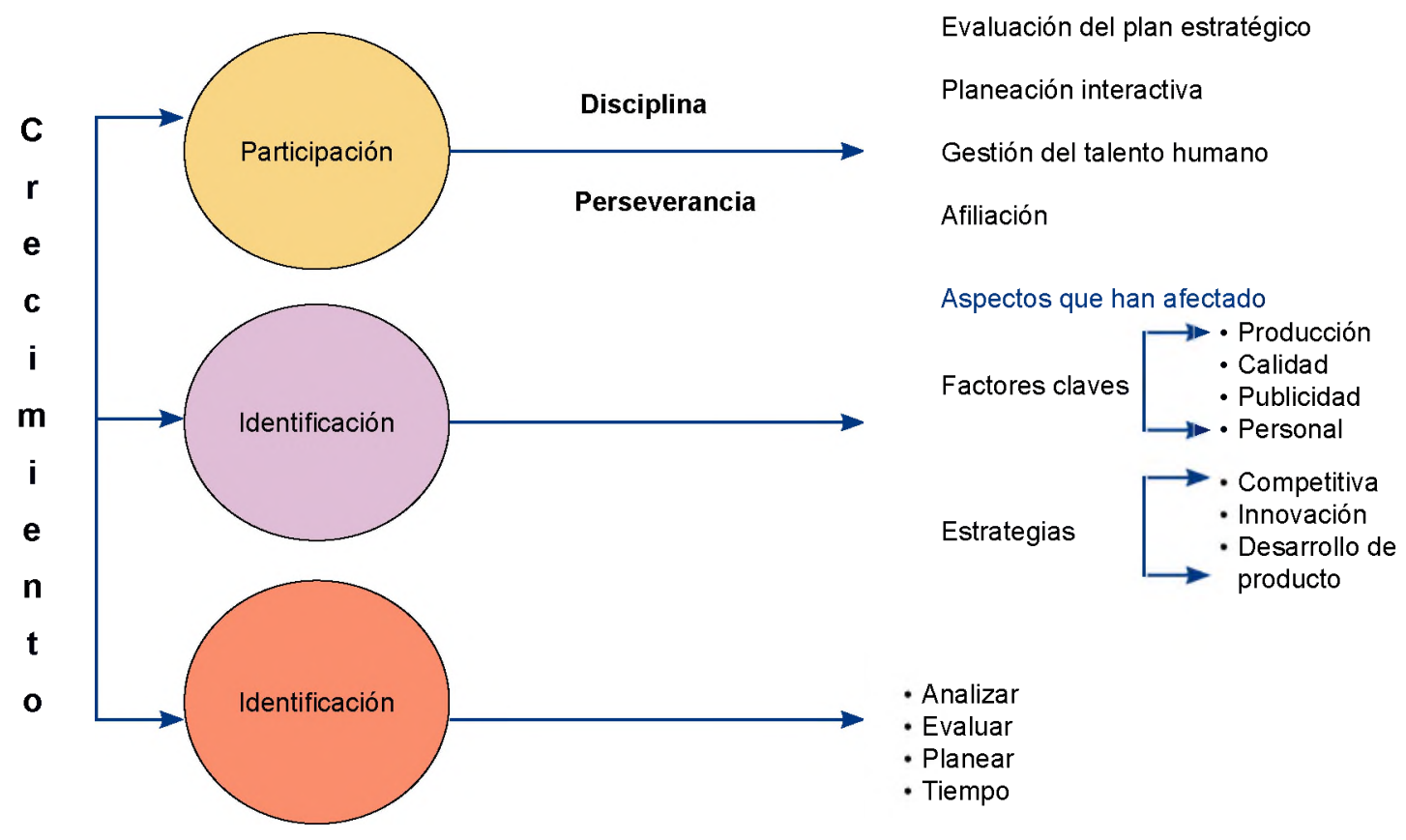

Figura 12. Modelo de factores claves de éxito: etapa de crecimiento.

\section{Conclusiones}

El sector de alimentos y bebidas es el más representativo en cuanto al aporte de la producción en la industria nacional, razón por la cual se tomó como subsector de referencia en la investigación. Este subsector ha logrado mitigar las amenazas de un mercado cambiante gracias a su crecimiento. Las empresas nuevas en este sector enfrentan diferentes barreras que impiden la puesta en marcha de sus proyectos y que en algunos casos obligan al cierre prematuro de estas organizaciones (pequeñas empresas, principalmente). Algunos factores que frenan su crecimiento son: poca financiación, costos elevados en materias primas, competencia desleal frente a la cadena de suministro, aumento en la legislatura de normas y el incremento y creación de nuevos impuestos.

En este análisis se observó como las PyME prestan mucha atención a sus trabajadores y a la selección de los mismos, debido a que estos son los que ejecutan las actividades del proceso y están encargados del buen funcionamiento de cada eslabón de la cadena de la organización. En su gran mayoría, las empresas no cuentan con certificaciones de calidad, lo que afecta en algún momento su crecimiento, ya que algunos contratos (ya sean de proveedores o clientes) exigen certificaciones para el cierre del negocio, debido a diferentes políticas de calidad que manejan las empresas.

Los factores claves que se deben tener en cuenta dependen principalmente de la organización y de las coyunturas en el tiempo, consecuencia de los cambios constantes que presentan los mercados 
y al tipo de enfoque que tenga cada empresa. Se debe resaltar que estos factores identificados se deben adaptar de acuerdo al entorno y al sector en el cual se encuentra inmerso una PyME. Finalmente, se recomienda a las empresas realizar cada una de sus estrategias, enfocándose en los factores claves de éxito y en el cumplimiento de sus objetivos y metas, así como tener disciplina y perseverancia en cada una de las actividades y proyectos en marcha.

\section{RefEREnCias}

ANIF. (2014). La gran encuesta PyME. Recuperado de http://anif.co/sites/default/files/uploads/ Gran\%20Encuesta\%20Pyme\%20II-2014_0.pdf

Cámara de Comercio de Bogotá. (2015). Tablero de indicadores de Bogotá y Cundinamarca 2014 No. 4. Recuperado de http://bibliotecadigital.ccb.org.co/handle/11520/13185

Cantillo, D. (2011, 17 de julio). Un país de PyMES. El Espectador. Recuperado de http://www. elespectador.com/noticias/economia/un-pais-de-pymes-articulo-285125

Guía del Emprendedor. (2015). Cómo hacer un modelo de negocio. Recuperado de bttps://www. lancetalent.com/blog/guia-del-emprendedor-modelo-de-negocio/

Ministerio de Industria y Comercio. (2015). Informe de industria a enero de 2015. Recuperado de http://www.mincit.gov.co/loader.php?1Servicio=Documentos\&lFuncion=verPdf\&id=7729 5\&name=Informe_de_Gestion_2015_A.pdf\&prefijo=file

Rockart, J. (1980). Los altos directivos definen sus necesidades de información. Recuperado de https:// es.scribd.com/doc/246124180/Lect-5-Factores-Criticos-del-Exito-Por-John-F-Rockart-pdf

Sesento, L., (2016). Modelo sistémico basado en competencias para instituciones educativas públicas. Recuperado de http://www.eumed.net/tesis-doctorales/2012/1sg/russel_ackoff.html

Superintendencia de Sociedades. (2015). Sector de alimentos y bebidas incrementó sus ingresos operacionales en 10\% durante 2014. Recuperado de http://www.supersociedades.gov.co/noticias/ Paginas/2015/Sector-de-alimentos-y-bebidasincrement\%C3\%B3-sus-ingresos-operacionales-en-10-durante-2014.aspx

Varela R., y Bedoya, O. (2006). Modelo conceptual de desarrollo empresarial basado en competencias. Estudios Gerenciales, 22(100). Recuperado de http://www.scielo.org.co/scielo.php?pid=S0123592320060003000018xcript=sci_arttext 
